***Reprinted with permission. No further reproduction is authorized without written permission from the Human Kinetics. This version of the document is not the version of record. Figures and/or pictures may be missing from this format of the document.***

\title{
Psychophysiological Response to Severe Sport Injury Among Competitive Male Athletes: A Preliminary Investigation
}

\author{
Renee Newcomer Appaneal, ${ }^{1}$ Frank M. Perna, ${ }^{2}$ \\ and Kevin T. Larkin ${ }^{3}$ \\ 1University of North Carolina, Greensboro; ${ }^{2}$ Boston University School \\ of Medicine; ${ }^{3}$ West Virginia University
}

\begin{abstract}
Features of posttraumatic distress have been associated with treatment noncompliance and delayed surgical recovery among general medical and trauma populations. Although cognitive-affective and behavioral features of posttraumatic distress have been demonstrated among adult and adolescent athletes with injuries, physiological responses associated with posttraumatic distress have not yet been examined in this population. The objective of this study was to examine psychophysiological stress reactivity to orthopedic trauma among male athletes who sustained a severe sport injury. Athletes with injuries $(n=7)$ and non-injured athlete controls $(n=5)$ completed self-report measures of psychological distress and were then shown injury video footage while heart rate and skin conductance measures were recorded. After exposure to orthopedic trauma-related video footage, athletes with injuries demonstrated significantly greater skin conductance reactivity and subjective distress compared to controls. As demonstrated among other medical and trauma populations, athletes with injuries exhibit exaggerated stress reactivity profiles when primed with orthopedic trauma stimuli.
\end{abstract}

Key Words: psychological distress, physiological reactivity, posttraumatic/acute stress, orthopedic trauma, psychology of sport injury

Despite improvements in conditioning methods, playing surfaces, and equipment, an estimated 3-17 million athletic injuries occur annually in the United States (Conn, Annest, \& Gilchrist, 2003). Approximately half of all high school and college athletes and up to $86 \%$ of football players will experience an athletic injury requiring treatment and prohibiting participation in athletic contests (Tunick, Etzel, Leard, \& Lerner, 1996). Figures from the National Collegiate Athletic Association (NCAA) indicate that the percentage of severe injuries is as high as $43 \%$ and $30 \%$ for popular sports such as football and women's

\footnotetext{
${ }^{1}$ University of North Carolina, Greensboro, Greensboro, NC 27402, E-mail: rrnewcom@uncg.edu; ${ }^{2}$ Boston University School of Medicine, Boston, MA 02118, E-mail: fperna@ bu.edu; ${ }^{3}$ Dept of Behavioral Medicine \& Psychiatry, West Virginia University, Morgantown, WV 26506, E-mail: kevin.larkin@ mail.wvu.edu.
} 
basketball, respectively, and surgery may be required to treat $15-20 \%$ of severe injuries incurring in those sports (NCAA, 1996). In studies examining postinjury psychological responses, athletes were commonly found to have elevated levels of negative affect, such as anger, tension, and in particular, depressed mood (Brewer, Petitpas, Van Raalte, Sklar, \& Ditmar, 1995; Leddy, Lambert, \& Ogles, 1994; Roh, Newcomer, Perna, Stilger, \& Etzel, 1998). In several cases, psychological distress reached severe levels, and rates of clinical depression ranged from 8-26\% (Brewer et al., 1995; Brewer, Linder, \& Phelps, 1995; Perna, Roh, Newcomer, Stilger, \& Etzel, 1998). Heightened negative affect has also been associated with decreased compliance with a variety of medical self-care procedures (Heatherton \& Renn, 1995) and specifically with reduced adherence to sport injury rehabilitation (Brewer et al., 2000; Daly, Brewer, Van Raalte, Petitpas, \& Sklar, 1995).

Although sport injury may be considered a stressful medical trauma, few researchers have explored athletes' post-injury responses using a biopsychosocial assessment approach like those that have been used for other commonly occurring traumatic events, such as motor vehicle accidents (MVAs) or receipt of medical diagnosis (Blanchard, Hickling, Barton, \& Taylor, 1996; Kiecolt-Glaser, Page, Marucha, MacCallum, \& Glaser, 1998). The American Psychiatric Association's Diagnostic and Statistical Manual-IV Text Revision (DSM-IV-TR) delineates categories of traumatic stress reactions (i.e., acute/posttraumatic stress disorder) where symptomatology clusters are typically expressed in cognitive-affective, behavioral, and physiological domains. For example, posttraumatic stress disorder (PTSD) represents a clinical reaction evidenced by a history of exposure to a traumatic event and the presence of symptoms across three areas that include persistent re-experiencing of the event (i.e., in the form of images, dreams, etc.), emotional numbing (i.e., staying away from reminders of it, trying not to think about it, etc.), and physiological arousal (i.e., motor restlessness, hypervigilance, etc.) (APA, 2000). The last two criteria for PTSD involve the duration of these symptoms (i.e., 1 month) and functional impairment in social or occupational domains (APA, 2000).

Although early trauma research examined posttraumatic reactions to severe events (e.g., war, violent crime, etc.), recent efforts have identified traumatic reactions in response to common events (e.g., motor vehicle accident, medical diagnosis, etc.). Among the general medical literature, victims of MVAs commonly report features of posttraumatic distress suggesting that events that occur with regular frequency may still pose a threat to a person's well-being (Blanchard et al., 1996; Blanchard, Hickling, Barton, Taylor, \& Loos, 1994; Bryant \& Harvey, 1996; Kuch, Cox, \& Evans, 1996; APA, 1997). In fact, the DSM-IV-TR identifies that in order for someone to meet the first criterion (i.e., trauma history), the person must have been exposed to a traumatic event where he or she experienced, witnessed, or had been confronted with an event or events that involve actual or threatened death or serious injury, or a threat to the physical integrity of oneself or others, as well as responded with intense fear, helplessness, or horror (APA, 2000). Because severe sport injury may threaten athletes' physical integrity and incidence rates are comparable to those of other commonly occurring traumatic events (i.e., MVAs), posttraumatic distress may have a unique application to psychology of sport injury and rehabilitation. 
Recent literature has documented that at least a few features of posttraumatic distress are present among athletes with injuries. For example, intrusive thoughts and avoidance behaviors have been found among both adult and adolescent athletes across gender, injury type (acute and chronic), and different sports (Newcomer \& Perna, 2003; Peck, Robertson, \& Zeffert, 1996; Shuer \& Dietrich, 1997). Moreover, Impact of Event Scale (IES) (Horowitz, Wilner, \& Alvarez, 1979) scores of athletes with severe sport injuries resemble those of MVA victims at 1 month post-injury (Newcomer \& Perna, 2003). It is noteworthy that athletes' elevated levels of posttraumatic distress may be prolonged (Peck et al., 1996) and triggered upon return to the sporting environment (Newcomer \& Perna, 2003). These findings suggest that athletes continue to experience emotional distress despite having physically recovered from injury, and the ongoing nature of athletes' injury-related distress is consistent with reports from other medical populations of prolonged emotional disturbance despite physical recovery from an event (Baum, 1990; Baum, Cohen, \& Hall, 1993; Blanchard et al., 1997; Holbrook, Anderson, Sieber, Browner, \& Hoyt, 1998; Kiecolt-Glaser et al., 1998). In addition to cognitive-affective and behavioral symptoms, elevated physiological arousal is also a core feature of posttraumatic distress and accompanies a variety of traumas including physical injury resulting from MVAs (Blanchard et al., 1996; Bryant, Harvey, Gordon, \& Barry, 1995; Orr, Lasko, Metzger, \& Pitman, 1997; Yehuda, McFarlane, \& Shalev, 1998). Similar to MVAs that are common yet may cause considerable psychophysiological distress, it is possible that athletes with injuries also experience physiological hyperarousal.

Because severe athletic injury may produce acute stress symptoms of similar magnitude to that of other traumatic stressors (e.g., MVAs) and because an athlete's injury may be recorded on videotape, examination of athletes' post-injury response may provide researchers with a unique opportunity from which to examine response to physical trauma that is not available with other traumatic injury groups (e.g., MVA victims). Similarly, the availability of non-injured athletes videotaped in the same environment also provides a level of control that is not commonly available in exposure studies with accident victims.

The purpose of our research was to use a traumatic-stress reactivity paradigm to examine athletes' post-injury reactions. Our primary aim was to determine athletes' psychophysiological reactivity to injury-related video stimuli. Specifically, we expected athletes with injuries, as compared to non-injured controls, to exhibit greater stress reactivity and that these between-group differences would become more evident as exposure to stimuli moved from non-injury (i.e., neutral) to more injury-related material (i.e., footage of athlete's actual injury).

\section{Methods}

\section{Participants}

This research was approved by institutional review boards at both universities where participants were recruited. Based on recent injury history and availability of injury video footage, 20 male participants from two NCAA Division I-II football teams were considered eligible for participation. Specifically, 10 athletes who had sustained a severe injury within the last 18 months that prevented participation for at least 1 month were referred to the study by the sports medicine staff. Control 
subjects $(n=10)$ were randomly selected from a stratified pool of athletes based on absence of a severe injury history and similarity to the athlete with injury (i.e., sport team, starting status, age, and race), and then matched pairs (i.e., athlete with injury and control) were contacted for participation.

Among the 20 eligible participants, 6 athletes were unavailable (e.g., graduated, did not return phone calls, etc.) and did not participate in the study, leaving a total of 14 participants who completed the study ( 7 injured and 7 control athletes). Two non-injured control athletes were dropped from the analyses after one athlete was erroneously categorized as a healthy control (i.e., sport medicine staff was unaware of recent injury) and another athlete's physiological data was invalid (i.e., signal interference). Twelve male athletes ( 7 injured and 5 controls) who were all starters for their respective teams and ranged in age from 19-25 years $(M=21.7$, $S E=0.50)$ completed the study. The sample was mostly African American $(75 \%)$ which is comparable to NCAA collegiate football participation rates for ethnic minorities; the remaining participants were Caucasian, not Hispanic.

To classify participants' injuries, two certified athletic trainers reviewed participants' medical history and rated current injury severity and overall injury history severity on a 4-point scale (none $=0$, mild $=1$, moderate $=2$, severe $=3$ ) based on criteria established by the American Academy of Orthopedic Surgeons (AAOS). Six of the seven athletes with injuries had incurred severe injuries and one had incurred a moderately severe injury within the past 18 months. With regards to prior injury history, six of the seven athletes had previously incurred injuries that were at least of moderate severity and one athlete with injury had no prior injury history. Among control athletes, only one of the five control athletes had a prior injury history, which was rated by a certified athletic trainer as severe.

At the time of enrollment (i.e., athletes' off-season), six of the seven athletes with injuries had been medically cleared to return to sport participation, and four had resumed play. The remaining three athletes were awaiting the start of the next competitive season to return to participation. Athletes with injuries had missed an average of 136 days $(S E=59.3)$ due to their most recent injury, and nearly 1 year $(M=353$ days, $S E=54.6)$ had lapsed since the date of their injury to the time of study enrollment.

\section{Procedure}

After completing a battery of self-report questionnaires, athletes were invited to an experimental room in a psychophysiology laboratory, electrodes were positioned, and they were instructed to rest for a 10-min habituation phase prior to stimulus presentation. After the laboratory phase, athletes were debriefed and provided with an opportunity to discuss any discomfort experienced as a result of their participation. Follow-up phone calls were made to all participants to determine presence of longterm adverse effects. These calls occurred at the end of the spring academic term when participants may have already left for summer vacation. However, successful contact by the researcher (i.e., first author) was made with $42 \%$ of participants; all reported no ill effects from their participation in this research.

Stimulus Presentation. The stimulus presentation consisted of four 3-min video segments each proceeded by a 3-min rest period and followed by a 3-min recovery period. The four segments consisted of edited video footage of four different scenarios: 
(1) the athletic injury (AI) occurrence of each recently injured participant, (2) a sportrelated (SR) clip free of any depiction of an athletic injury, (3) a general injury (GI) clip depicting an injury sustained by a professional athlete-actor in the same sport as participants, and (4) a control/neutral (NE) segment of a local scenic landscape. The athletic injury segment included the play in which the athlete's injury occurred and its aftermath (e.g., athletic trainer evaluation, removal from the field, etc.), whereas the sport-related segment consisted of athlete's team footage unassociated with injury. Both the athletic injury and sport-related segments depicted the athlete with the injury's competitive environment and contained video footage of athletes participating in the study that was comparable to what would be shown during a televised sporting event. A football injury sequence from a major motion picture (i.e., Jerry McGuire) was used for the general injury segment, and the neutral stimulus consisted of a non-injury, non-sport, scenic nature still shot. Three of the four video segments (AI, SR, and GI) were $1.5 \mathrm{~min}$ in duration and were repeated once, resulting in an overall 3-min video segment. Injury-related video footage (athletic and general injury clips) included the preceding play at normal speed, the injury occurring in slow motion, and on-field or sideline shots at normal speed. Control athletes were shown the athletic injury segment for the athlete with injury that they were matched to for recruitment into the study. Lastly, to minimize order effects, each athlete pair (injured and control) was randomly assigned to 1 presentation order from 12 possible order combinations.

\section{Measures}

Physiological Reactivity. Physiological reactivity to stimulus presentation was measured by heart rate (HR) and two measures of electrodermal activity (EDA): skin conductance level (SCL) and skin conductance response (SCR). All physiological reactivity variables were measured using disposable MediTrace pellet electrodes (Tyco Healthcare Group, Mansfield, MA). A bipolar placement centered on the medial phalanx of participants' non-dominant fore and middle fingers was used for EDA, and HR was measured by three electrodes placed on participants' right and left shoulders and left hip. HR signals were amplified using a Grass model 7P4F pre-amplifier (Grass Instruments, Quincy, MA), and EDA signals were amplified using a Grass model 7P1DE pre-amplifier. Signals were recorded continuously over each 9-min sequence (rest, stress, and recovery periods) and transmitted from the Grass polygraph to an IBM-compatible computer with DATAQ software (DATAQ Instruments, Inc., Akron, OH).

HR was expressed as the average beats per minute (bpm). For EDA, resistance to a constant current was measured continuously over the course of the experimental session (i.e., as palmer sweat index increased, resistance was lowered). Electrical resistance was transformed to skin conductance level (SCL), converted from an analog to digital form, and expressed in microsiemens $(\mu S)$ for each period (Andreassi, 2000). SCR was defined as the number of momentary upward fluctuations that occurred during each segment condition that met or exceeded an amplitude criterion of either $0.1 \mu \mathrm{S}$ for SCL ranging between $0-10 \mu \mathrm{S}$ or $0.2 \mu \mathrm{S}$ for SCL above $10 \mu \mathrm{S}$ (Andreassi, 2000). Relatively higher EDA values indicated relatively greater autonomic activity.

Subjective Reactivity. Participants' subjective reactivity to stress segments (AI, $\mathrm{SR}, \mathrm{GI}$, and NE) was measured using two brief self-report scales administered 
following recovery and prior to subsequent rest period for each stress segment. Participants completed a six-item version of the Profile of Mood States (POMS) (McNair, Lorr, \& Droppleman, 1971) referred to as the Brief Assessment of Mood (BAM) (Dean, Whelan, \& Meyers, 1990). The BAM is a self-report instrument with six mood state items (tension, anger, depression, vigor, confusion, and fatigue), reflective of the six POMS subscales. In a large validity study with collegians $(N=621)$, Dean et al. reported acceptable correlations between BAM items and POMS counterpart scales ( $r$ values $=.66$ and .87 , respectively) (Dean et al., 1990). The BAM was also correlated with autonomic activity (e.g., cortisol response) in a controlled study with collegiate athletes (Perna, Antoni, Baum, Gordon, \& Schneiderman, 2003). Because prior studies suggested that depression, anger, and tension were the mood states that most clearly distinguish between athletes with and without injuries (Lavallee \& Flint, 1996), an index of Negative Affect in response to each video segment (BAM-NA) was derived by a sum of the three negative mood items (i.e., tension, anger, and depression). For Subjective Units of Distress Scale (SUDS) (Stone, 1997), athletes marked along a 15-cm visual analog scale how the preceding video segment (AI, SR, GI, or NE) made them feel (anchors: Not at all upset and Extremely upset). Lastly, as part of debriefing, athletes ranked the four video segments from 1 to 4 ( 1 = least distressing and $4=$ most distressing).

Background Psychological Distress. Because preexisting psychological distress might influence physiological reactivity, athletes' self-reported level of psychological distress at the time of study participation was measured using a battery of questionnaires. The survey packet was administered prior to the laboratory-based reactivity portion of the study and included measures of posttraumatic distress, negative affect, and life-event stress.

Posttraumatic distress was measured using the Impact of Events Scale (IES) (Horowitz et al., 1979). The IES is a widely used and highly reliable 15-item selfreport instrument designed to assess intrusive thoughts and avoidance behaviors (subscales), and it has been used among a variety of athlete, medical, and trauma populations (Baider \& De Nour, 1997; Delahanty, Herberman, Craig, Hayward, et al., 1997; Ironson et al., 1997; Newcomer \& Perna, 2003). Respondents were asked to consider their most recent serious athletic injury and report the degree $(0=$ not at all, $1=$ rarely, $3=$ sometimes, $5=$ often $)$ to which they experienced intrusive thoughts and engaged in avoidance behaviors within the past 2 weeks. As each participant was asked to consider his injury history, it was assumed that all athletes experience physical injury of varying severity (i.e., muscle soreness to torn ligaments) regardless of whether their injuries were severe enough to warrant medical attention. In fact, it is intended to evaluate the impact of an injury that the athlete, not the medical staff, considers significant. Psychometric evaluations of the IES report acceptable levels of internal consistency and 2-week test-retest reliability of .78-.82 and .79-.89 for the intrusive thoughts and avoidance behavior subscales, respectively (Zilberg, Weiss, \& Horowitz, 1982).

Negative affect was measured using a shortened POMS (Shacham, 1983). The shortened POMS is a 37-item version of the POMS (McNair et al., 1971), and administration instructions require respondents to consider how they have felt over the past week including today. Similar to BAM-NA, an index of Negative Affect was derived by a sum of the three negative mood scales (i.e., tension, anger, and depression) from the shortened POMS. 
Life-event stress was measured using the Life-event Scale for Collegiate Athletes (LESCA) (Petrie, 1992). The LESCA has been prospectively related to athletic injury incidence, cortisol level, and symptoms suggestive of heightened autonomic nervous system (ANS) activity among athletes (Perna \& McDowell, 1995; Petrie, 1993, 1993).

\section{Statistical Analyses}

As posttraumatic distress, negative affect, life-event stress, and physiological arousal (HR and EDA) have been identified as having potential confounding effects on both physiological and psychological reactivity (Cacioppo, 1994; Pike et al., 1997), potential a priori between-group differences were examined using a series of independent samples $t$ tests. Although a recovery condition was included, participants' physiological arousal may have changed from one pre-segment rest period to the next, which may have affected subsequent changes in physiological reactivity to stress segments. Therefore, change across rest periods was analyzed using three 2 [group (injured, control)] $\times 4$ [rest period $(1,2,3,4)]$ mixed factors ANOVAs with mean SCL, SCR, and HR as dependent measures.

An a priori planned contrast analysis approach was used to test the hypothesis that reactivity among athletes with injuries would be greater than non-injured athletes' reactivity to all segments, and the magnitude of this between-group difference would become greater as exposure to stimuli changed from neutral material (i.e., $\mathrm{NE}$ and SR segments) to more injury-related material (i.e., GI and AI segments) where differences in physiological reactivity between groups would be most evident during the specific injury segment (i.e., AI). A planned contrast approach is considered to be superior to ANOVA because it provides a more focused analysis $(1 d f F)$ than an omnibus test $(F d f>1)$, and decreases the likelihood of Type II error while maintaining the same probability of Type I error as an omnibus ANOVA (Rosenthal \& Rosnow, 1988).

First, three 2 [group (injured, control)] $\times 4$ [stimuli (AI, GI, SR, NE)] mixed factors ANOVAs were calculated to obtain mean square error terms for use in the planned contrast analysis. Then, non-orthogonal lambda $(\Lambda)$ weights were assigned according to the hypothesis that the magnitude of difference between injured and non-injured athletes' physiological responses (PR) would become greater as exposure to stimuli changed from neutral material (i.e., NE and SR segments) to more injury-related material (i.e., GI and AI segments) where differences in PR between groups would be most evident during the specific injury segment (i.e., AI). Last, three 2 (group: injured, non-injured) $\times 4$ (stress segment: AI, GI, SR, NE) planned contrasts were conducted with HR, SCL, and SCR as dependent measures and covarying for participants' baseline physiological level (Rosenthal \& Rosnow, 1988). The same pattern of non-orthogonal $\Lambda$ weights was also applied in planned contrasts analyses for participants' subjective reactivity (i.e., SUDS and BAM-NA).

A Mann-Whitney analysis was conducted to compare rankings per segment between athletes with and without injuries. A Friedman test was used to compare overall rankings across segments, and three Wilcoxon analyses were conducted as follow-up comparisons to examine rank given to AI as compared to GI, SR, and NE segments. 


\section{Results}

\section{Preliminary Analyses and Data Reduction}

Of the 12 athletes who participated, only 11 ( 7 injured and 4 non-injured) had valid EDA data, whereas all 12 athletes ( 7 injured and 5 non-injured) had valid self-report and HR data. Independent-samples $t$ tests revealed no significant difference between controls and athletes with injuries in life-event stress $(M \mathrm{~s}=9.60$ and 9.86, SEs $=$ 4.13 and 3.45, respectively) or POMS-Negative Affect $(M \mathrm{~s}=13.60$ and 9.86, SEs = 4.80 and 3.17, respectively), $t$ values $(10)=0.05$ and 0.65 , respectively, $p$ values $>$.05. Furthermore, mean IES intrusion and avoidance scores were not significantly different among controls $(M \mathrm{~s}=2.80$ and 4.20, $S E \mathrm{~s}=1.56$ and 2.56, respectively) and athletes with injuries $(M \mathrm{~s}=1.71$ and 3.29, $S E=1.08$ and 1.66 , respectively), $t$ values $(10)=0.57$ and 0.30 , respectively, $p$ values $>.10$.

Although physiological arousal generally increased over time for all participants, only a significant time effect was found for SCL, $F(3,27)=17.0, p<.001$, and not for SCR or HR, $F$ values $(3,27)=0.80$ and 0.48 , respectively, $p$ values $>.10$. As a result, athletes' SCL was adjusted for this time-effect when calculating change scores.

Average HR and EDA levels were computed for the habituation phase and across each of the four rest and subsequent stress conditions (i.e., AI, GI, SR, and NE). Consistent with prior suggestions (Llabre, Spitzer, Saab, Ironson, \& Schneiderman, 1991), delta $(\Delta)$ scores were calculated for HR and EDA. Specifically, participants' habituation baseline was used to calculate $\Delta$-HR and $\Delta$-SCR, whereas $\Delta$-SCL was calculated by subtracting pre-condition rest periods from average SCL during stimulus presentation segments covarying for pre-condition baseline.

\section{Physiological Reactivity}

Participants' physiological arousal during rest and subsequent stress conditions is provided in Table 1 . The hypothesized trend was found for $\Delta$-SCL, $F(1,24)=$ $16.04, p<.001$, but only marginally supported for $\Delta$-SCR, $F(1,24)=3.74, p<.10$, and not supported for $\Delta-\mathrm{HR}, F(1,27)=1.88, p>.10$ (refer to Figure 1). As seen in Figure 1, athletes with injuries exhibited greater physiological reactivity than non-injured controls, and the greatest between-group difference was clearly evident during AI stimuli. Athletes' physiological reactivity to GI and SR stimuli, compared to the AI stimuli, was minimal, and group's reactivity patterns across EDA and HR measures were quite similar during these segments.

\section{Subjective Reactivity}

The hypothesized trend between groups and across stimuli was also supported for both SUDS and BAM-NA, $F$ values $(1,30)=9.77$ and $16.94, p$ values $<.01$ and .001 , respectively (see Figure 2). Similar to physiological reactivity, subjective reactivity among athletes with injuries was higher than controls, and the greatest between-group difference was found in response to AI stimuli.

Overall, only two of the five planned contrast analyses of reactivity to stimulus presentations did not reach the .05 level of statistical significance (see Table 2). However, as illustrated in Table 2, the effect sizes for both $\Delta$-SCR and $\Delta$-HR were large $(d$ values $=1.34$ and .88 , respectively $)$. 
Table 1 Mean Skin Conductance Level (SCL) and Response (SCR), and Heart Rate (HR) Across Four Pre-condition Baselines and Stress Segments for Injured and Control Groups*

\begin{tabular}{lcccccc}
\hline & \multicolumn{2}{c}{ SCL $(\mu \mathrm{S})$} & \multicolumn{2}{c}{ SCR } & \multicolumn{2}{c}{ HR (bpm) } \\
\cline { 2 - 7 } Segment & Injured & Control & Injured & Control & Injured & Control \\
\hline \multicolumn{1}{c}{$\boldsymbol{N}=$} & $\mathbf{7}$ & $\mathbf{4}$ & $\mathbf{7}$ & $\mathbf{4}$ & $\mathbf{7}$ & $\mathbf{5}$ \\
\hline Baseline & $4.76(1.27)$ & $7.55(2.26)$ & $1.96(0.92)$ & $3.75(1.07)$ & $62.76(4.03)$ & $73.41(3.56)$ \\
Athletic & & & & & & \\
injury & $7.15(1.43)$ & $7.55(2.22)$ & $5.67(1.42)$ & $3.83(0.96)$ & $63.83(4.17)$ & $71.94(2.44)$ \\
Baseline & $4.74(1.10)$ & $6.06(1.63)$ & $2.70(0.81)$ & $3.26(1.12)$ & $62.76(4.03)$ & $73.41(3.56)$ \\
General & & & & & & \\
injury & $4.83(1.26)$ & $6.32(2.91)$ & $2.38(0.76)$ & $3.42(1.61)$ & $62.49(4.27)$ & $74.61(3.99)$ \\
Baseline & $5.29(1.20)$ & $6.98(2.16)$ & $2.27(0.73)$ & $2.70(0.47)$ & $62.76(4.03)$ & $73.41(3.56)$ \\
Sport-only & $5.91(1.44)$ & $7.47(2.33)$ & $3.24(1.27)$ & $4.83(1.34)$ & $64.80(3.87)$ & $73.38(2.35)$ \\
Baseline & $5.06(1.43)$ & $7.24(2.73)$ & $1.71(0.72$ & $2.78(0.81)$ & $62.76(4.03)$ & $73.41(3.56)$ \\
Neutral & $4.33(1.27)$ & $6.26(2.89)$ & $1.57(0.73)$ & $2.50(1.19)$ & $67.69(3.23)$ & $77.76(4.30)$ \\
\hline
\end{tabular}

Note. Standard error (SE) is given in parentheses.
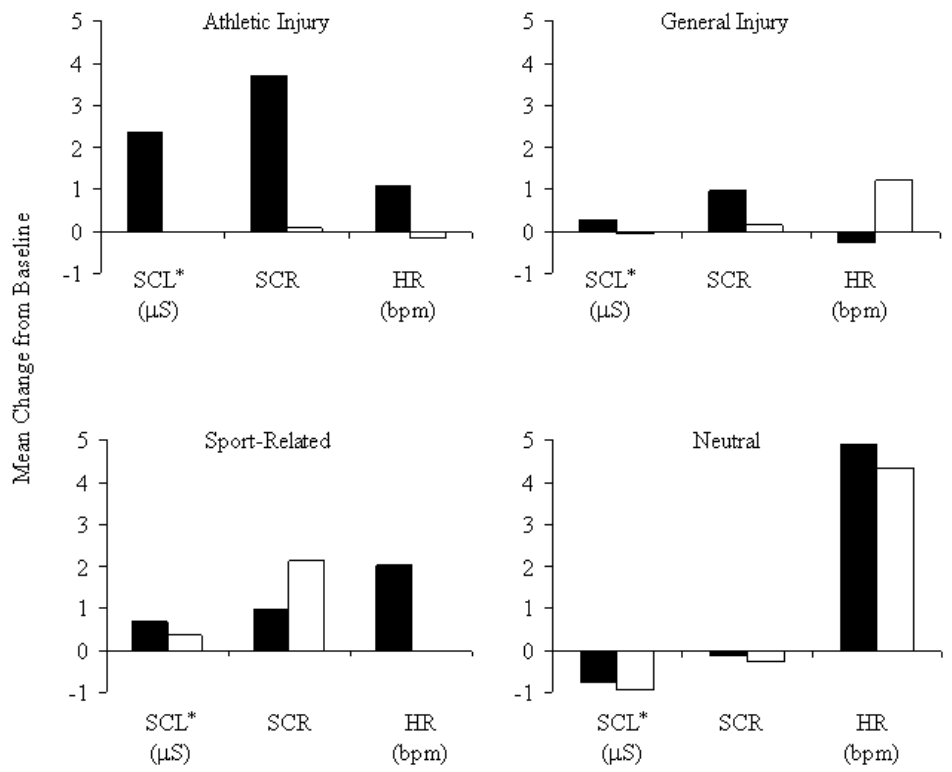

Figure 1-Injured $(n=7)$ and non-injured athletes' $(n=4,5)$ mean change from baseline in physiological reactivity (adjusted SCL, SCR, and HR) across four segment presentations. *Adjusted for baseline differences in SCL. *A planned contrast analysis revealed a significant contrast trend for $\Delta \mathrm{SCL}, F(1,24)=16.04, p<.001$, but not for $\Delta \mathrm{SCR}, F(1,24)$ $=3.74, p<.10$, or $\Delta \mathrm{HR}, F(1,27)=1.88, p>.10$. 
Table 2 Effect Sizes for A Priori Planned Contrast Analyses of Participants' Subjective Distress and PR to Stress Segments

\begin{tabular}{llllll}
\hline $\begin{array}{l}\text { Dependent } \\
\text { measure }\end{array}$ & $\boldsymbol{N}$ & $\boldsymbol{F}$ & $\boldsymbol{d} \boldsymbol{p}$ & $\boldsymbol{p}<$ & Cohen's $\boldsymbol{d}$ \\
\hline$\Delta-\mathrm{SCL}^{\mathrm{a}}$ & 11 & 16.04 & 1,24 & .001 & 2.77 \\
$\Delta-\mathrm{SCR}^{\mathrm{b}}$ & 11 & 3.74 & 1,24 & .10 & 1.34 \\
$\Delta$-HR $^{\mathrm{b}}$ & 12 & 1.88 & 1,27 & .20 & .88 \\
SUDS $^{\mathrm{a}}$ & 12 & 9.77 & 1,30 & .01 & 2.01 \\
BAM-NA $^{\mathrm{a}}$ & 12 & 16.94 & 1,30 & .001 & 2.64 \\
\hline
\end{tabular}

${ }^{\text {aS }}$ Statistically significant at $p<.05$ level.

${ }^{\mathrm{b}}$ Non-significant.

Table 3 Participants' Mean Ranking of Stress Segments*

\begin{tabular}{ccccccc}
\hline Segment & $\boldsymbol{M}(\boldsymbol{S E})$ & & Segment & $\boldsymbol{M}(\boldsymbol{S E})$ & $\mathbf{Z}$ & $\boldsymbol{p}<$ \\
\hline \multirow{2}{*}{ AI } & $3.83(.17)$ & vs. & GI & $2.42(.19)$ & -3.15 & .01 \\
& & vs. & SR & $2.42(.19)$ & -2.85 & .01 \\
& & vs. & NE & $1.33(.26)$ & -3.15 & .01 \\
\hline
\end{tabular}

*Friedman's $\chi^{2}(3, N=12)=22.70, p<.001$.

\section{Video Segment Rank}

Friedman's test of multiple dependent ranks revealed that segments were ranked significantly different, $\chi^{2}(3, N=12)=22.70, p<.001$. As reflected in physiological reactivity data, participants' subjective ratings indicated that the AI segment appeared to be the most distressing. As can be seen in Table 3, the AI segment was ranked significantly more distressing $(M=3.83, S E=.017)$ than the GI, SR, or NE segments $(M \mathrm{~s}=2.42,2.42$, and $1.33, S E \mathrm{~s}=0.19,0.19,0.26$, respectively $)$. A Mann-Whitney test revealed no significant between-group difference in rankings $[Z(3, N=12) p$ values $>.50]$.

\section{Discussion}

To our knowledge, no published research has directly examined athletes' physiological arousal (e.g., heightened ANS activity) associated with sport injury. The present study used a sport injury paradigm to examine psychophysiological reactivity to traumatic stimuli. We hypothesized that athletes with injuries, as compared to non-injured controls, would exhibit greater stress reactivity to video clips depicting injury-related and noninjury-related stimuli, and our hypothesis was supported for one physiological (i.e., SCL) and both subjective (i.e., SUDS and BAM-NA) measures of reactivity. It is noteworthy, however, that $\Delta$-SCL was statistically significant between 
Athletic Irjury

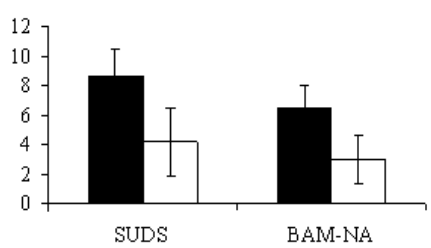

Sport-Rebated

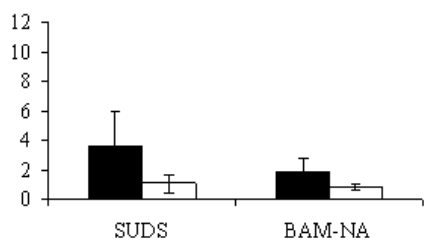

Injured
General Irjury

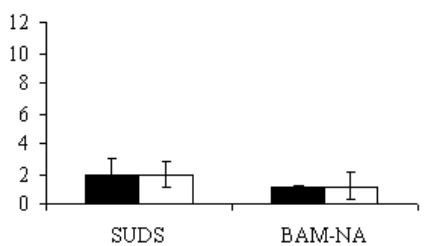

Neutral

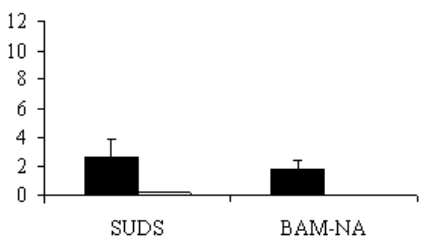

Non-injured

Figure 2-Injured $(n=7)$ and non-injured athletes' $(n=5)$ mean ratings of subjective reactivity (SUDS and BAM-NA) across four segment presentations. A planned contrast analysis revealed a significant contrast trend for SUDS and BAM-NA, $F$ values $(1,30)=$ 16.94 and $9.77, p$ values $<.001$ and .01 , respectively.

injured and non-injured groups, whereas the $\Delta$-SCR and $\Delta$-HR were not statistically significant ( $p$ values $=.10$ and .20 , respectively). The magnitude of effect sizes for physiological and psychological stress reactivity ( $d$ values ranged from .88 to 2.77 ) further suggests that there were divergent between-group patterns in stress reactivity to the stimulus presentation.

The divergence in physiological reactivity indices across the four video segments suggests that different behavioral processes (i.e., coping response) may have occurred. In fact, it has been well established that dysynchronous patterns for EDA and HR have been observed across different tasks (Fowles, 1988; Lacey, Kagan, Lacey, \& Moss, 1963). Originally proposed by Gray (1987), cortical arousal involves two separate but overlapping motivational systems that control behavior: behavioral activation (BAS) and behavioral inhibition (BIS). The behavioral activation system regulates approach behaviors most commonly associated with behaviors that result in reinforcement, whereas the BIS is involved in avoidance or escape behaviors most commonly associated with punishment. Fowles (1988) examined these two motivational systems using psychophysiological methods of measurement, and these findings have since been replicated (Heponiemi, Keltikangas-Jarvinen, Kettunen, Puttonen, \& Ravaja, 2004; Schwerdtfeger, 2004; Waldstein, Bachen, \& Manuck, 1997). HR appears to be a better index of the BAS or motivational approach and task engagement, whereas EDA appears to be more closely linked to the BIS or anxiety and avoidance (Fowles, 1988; Heponiemi et al., 2004). Consistent with 
Fowles' hypothesis, we observed a relation between EDA and injury-related stimuli (i.e., athletic injury clip), presumably evoking an avoidance response or inhibition. Increased reactivity to the AI segment among athletes with injuries is suggestive of active efforts to inhibit psychological distress (i.e., BIS) associated with their injuries that may have been elicited while viewing injury-related and personally relevant material that was upsetting. In fact, athletes' self-reported reactivity (i.e., SUDS and BAM-NA) to and ranking of the AI segment indeed suggest it was the most anxiety-provoking segment. BIS activation has also been conceptually associated with coping with distress, whereas BAS activation has been linked to coping without distress.

The only observed heightened HR responses to stimulus presentations for both athletes with and without injuries were to the neutral stimulus. Although the reason for this is uncertain, it can be hypothesized that the discrepancy in HR response was related to the video format of the three sports-related videos and the still image of the neutral footage. It is well known that video tasks that engender sensory intake are typically accompanied by small HR reactions or even reductions in HR (Lacey et al., 1963) in contrast to the exaggerated HR responses observed during mental tasks (i.e., sensory rejection tasks). Data from other psychophysiological studies indicate that a still motion image may elicit a different response pattern than film containing motion (Prins, Kaloupek, \& Keane, 1995). It is possible that the participants in this study became disinterested in the neutral scene and focused on some other mental task or image that evoked this HR response (i.e., they returned to their own thought process rather than to the image being depicted). Because the nature of this cognitive activity was not assessed, this is only speculation at this point.

Our data and the use of the athletic milieu are novel and contribute to the stress reactivity literature by offering natural compare/control conditions that typically are not available in posttraumatic stress research. According to the general trauma literature, physiological reactivity generally occurs in response to stimuli closely associated with the trauma itself (i.e., trauma specificity) rather than generic stressors (Casada, Amdur, Larsen, \& Liberzon, 1998). Unfortunately, a personally relevant visual trauma stimulus is nearly impossible to obtain for trauma victims typically involved in traumatic stress and reactivity research (e.g., combat veterans, victims of MVA or assaults, etc.). As a result, trauma researchers often use a generic stimulus that closely resembles the traumatic event studied (e.g., sounds or imagery scripts). However, in the present study, actual personally relevant trauma stimuli (i.e., athletic injury video) were available. Consistent with theory, athletes with injuries did not appear to consider the general injury stimuli as emotionally threatening, especially in comparison to viewing their own injuries. As illustrated in Figure 1, physiological reactivity to the GI compared to the personally relevant AI stimulus among athletes with injuries only reflected a minimal increase from baseline $(0.90$ and $2.39 \mu \mathrm{S}$, respectively). In terms of subjective reactivity (SUDS and BAM-NA), athletes with injuries rated the GI segment as the least anxiety-provoking stimulus, which suggests that athletes merely watched without anxiety to the GI segment.

An unexpected finding in the present study concerned the lack of betweengroup differences in posttraumatic distress on the self-report questionnaires (e.g., IES scores), especially considering that exaggerated psychophysiological reactivity to trauma stimuli among athletes with injuries as compared to controls was clearly evident. However, IES measures were taken prior to the stress 
reactivity laboratory session. Findings from our previous work suggest that the time of athletic season when our assessment was conducted for this study may have influenced athletes' self-reported injury-related distress (Newcomer et al., 1998). In the present study, athletes were recruited and assessed during the offseason, and during this time, athletes have the ability to control their exposure to injury-related stimuli. For example, both athletes with and without injuries are required to attend daily practices and team meetings during the pre- and regular seasons, whereas few requirements are imposed on them beyond the regular season. Subsequently, athletes who are experiencing psychological distress related to becoming injured must engage in active efforts to avoid stimuli associated with the injury during the regular season, which, in turn, may heighten intrusive thoughts (Davies \& Clark, 1998; Harvey \& Bryant, 1998; Purdon \& Clark, 1994; Shiperd \& Beck, 1999; Trinder \& Salkovskis, 1994; Wegner \& Zanakos, 1994). Specifically, thought suppression requires active efforts to avoid intrusive thoughts, and that effort actually facilitates the maintenance of the distressful intrusions. Repression, another avoidance strategy, is commonly used among trauma victims (Davis, 1990; Joseph, 1998; Ward, Leventhal, \& Love, 1988). Repressors attempt to maintain a socially favorable image and minimal levels of negative affect that are consistent with their self-concepts (Holmes, 1990; Weinberger, 1990). However, recent findings demonstrate that although repressors report low levels of distress, physiological indices (particularly skin conductance measures) suggest distress is present (Asendorpf \& Scherer, 1983; Holmes, 1990; Martin \& Pihl, 1986; Wickramasekera, 1994).

The "culture" of athletics encourages athletes to not talk about their fears, concerns, and level of distress regarding injury (Curry \& Strauss, 1994; Nixon, 1992, 1993, 1994). This environment, particularly prior to and during the competitive season, could actually prime athletes for avoidance strategies such as thought suppression and repression. However, during the off-season, self-reported distress may be minimized, which might suggest that physiological arousal might be a more accurate measure of injury-related distress. Our prior work (Newcomer et al., 1998; Newcomer et al., 2003) found that in comparison to controls, athletes with injuries experienced significant elevations in intrusive thoughts and avoidance behavior immediately post-injury and again during the immediate pre-season. During the off-season, self-reported injury distress may be lower because athletes who are injured or have recovered from a recent injury are not exposed to trauma-related stimuli (e.g., sporting venue, coaching staff, teammates, etc.) to the same degree as they would be during the regular season. In fact, our athlete sample did report generally low frequencies of intrusive thoughts and avoidance behaviors $(M \mathrm{~s}=1.71$ and $3.28, S E s=1.08$ and 1.66 , respectively). However, when primed by injuryrelated stimuli, athletes with injuries clearly exhibited heightened physiological (i.e., SCL) and psychological reactivity (i.e., SUDS and BAM-NA) as compared to controls. Lack of emotional processing of a traumatic event and hyperarousal are thought to be principally involved in the development of heightened traumarelated distress and possibly acute/posttraumatic stress disorder (Blanchard et al., 1996; Bryant \& Harvey, 1997; Harvey \& Bryant, 1998; Warda \& Bryant, 1998). The tendency to escape and avoid psychological distress, which has been collectively referred to as experiential avoidance (Hayes, Wilson, Gifford, Follette, \& Strosahl, 1996), may have important clinical implications for athletes coping with 
injury-related distress within a competitive sport culture that does not provide for emotional expression.

In conjunction with relevant findings, it is important that we acknowledge potential limitations of the present study, such as sample size and demographics, heterogeneous injury diagnoses, and the potentially unsuitable control stimuli (i.e., NE segment). With respect to sample size, the relatively small sample $(N=12)$ may have limited the power needed to detect significant changes in physiological response between groups and across stimulus presentation. However, the primary analyses yielded rather large effect sizes (Cohen's $d$ ranged from .88 to 2.77 ) that indicated there were divergent patterns of reactivity to the stimulus presentation between groups, which supported the primary hypothesis.

In addition to sample size, the large percentage of ethnic minorities (75\%) in our sample may have contributed to exaggerated reactivity response. Ethnic minority status has been associated with elevated cardiovascular reactivity (e.g., blood pressure, $\mathrm{HR}$, etc.) as well as increased risk for posttraumatic distress following a traumatic event (Dimsdale, 2000). It is possible that a reactivity pattern might have been less robust had we recruited an ethnically balanced sample. However, because relatively more African Americans participate in sports with a high injury risk (i.e., football) than non-contact sports (i.e., golf), we believe our sample was representative of the sport (NCAA, 2005). Unfortunately, due to the small sample size, ethnic minority status could not be examined as a between-subjects factor in the present study.

Despite athletes' injuries having been classified as severe in nature, another potential limitation in the present study was that athletes with injuries had sustained a variety of orthopedic traumas (e.g., career-ending cervical spine injury, torn knee ligament, etc.) as opposed to a homogeneous injury sample (i.e., anterior cruciate ligament injury). However, this is not unusual for trauma research where a variety of physical injuries may result from a single type of trauma event (i.e., MVAs), and it only highlights the need for researchers to use standard measures of injury severity. Similarly, injury severity in our study was determined by AAOS guidelines, which are comparable to the Injury Severity Score (ISS) used to triage emergency room patients and compare injuries among trauma victims (Greenspan, McLellan, \& Greig, 1985). Moreover, it is possible that prior injury history may moderate athletes' psychophysical reactivity to injury-related stimuli. Although this was beyond the scope of the current study, future research might explore the relationship between athletes' reactivity and extent injury history (i.e., no prior injury compared to multiple prior injuries).

In our study, physiological reactivity among athletes with injuries appeared to be greatest during stimuli associated with personally relevant trauma stimuli (i.e., AI) rather than to stimuli associated with a general injury that was not personally relevant. This finding is consistent with data from other trauma populations where the greatest degree of reactivity occurs during trauma-related stimuli (Casada et al., 1998; Shalev, Peri, Gelpin, Orr, \& Pitman, 1997). Considering that serious athletic injury is highly likely in certain sports (e.g., 15-20\% of sport participants) and that competitive sporting events are often videotaped (e.g., televised or coaches' video analysis), the access to personally relevant trauma stimuli provides a unique opportunity to examine assumptions of trauma specificity. The nature of the athletic environment (i.e., pre-injury screening and predictable injury rates) also provides an opportunity for researchers to obtain data regarding premorbid functioning as 
well as to select closely matched healthy controls, both of which facilitate a level of design control nearly impossible to achieve with other traumatic events (e.g., MVA, war, etc.).

Our findings and those of other sport injury researchers suggest that athletes with injuries resemble other medical populations in their psychophysiological response to trauma, which, in turn, demonstrates the relevance of sport injury research to general literature concerning response to physical health trauma. Exaggerated ANS activity may be particularly problematic for persons undergoing surgery to repair athletic injury. Delayed surgical wound healing has been demonstrated under periods of high stress (i.e., medical school exams and dementia care giving) (Kiecolt-Glaser, Marucha, Malarkey, Mercado, \& Glaser, 1995; Marucha, Kiecolt-Glaser, \& Favagehi, 1998). Moreover, it has been proposed that effects of heightened ANS activity on the immune system may underlie the link between psychological stress and impaired surgical wound healing (Kiecolt-Glaser et al., 1998). More than a decade of research exists examining the relationship between psychological stress and immune system functioning (see review by Kiecolt-Glaser, McGuire, Robles, \& Glaser, 2002), and sleep disturbance, which frequently accompanies emotional distress, has been identified as a possible mediator in the stress-immune relationship (Hall, et al., 1998). Considering that psychological stress-induced increases in autonomic activity have also been associated with prolonged recovery from exercise (Perna $\&$ McDowell, 1995) and that orthopedic rehabilitation often requires intense physical therapy, psychological sequelae associated with athletic injury may impair physical recovery and exercise training adaptation.

From our findings, athletes do seem to experience psychophysiological reactivity to sport injury. However, caution should be used in generalizing our findings to non-male or non-collegiate athlete samples. Future research should seek to include larger and more diverse athlete samples (e.g., ethnicity, sex, age, etc.) to examine potential individual differences in reactivity to sport injury. Considering the multidimensional nature of the stress response, future research examining athletes' post-injury reactions should include assessment approaches reflective of a biopsychosocial model. One such approach may be the use of psychophysiological reactivity. If psychophysiological recording equipment is not feasible, then researchers might consider using self-report measures that reflect cognitive-affective, behavioral, and physiological features of stress. For example, the Impact of Events Scale-Revised (Weiss, 2004), which was not available at the time of data collection in our study, includes three subscales reflective of the three symptom clusters of PTSD (i.e., intrusions, avoidance, and hyperarousal). Further study is needed to determine if athletic injury is truly appropriate as a model for other health-related traumatic stressors. Considering the methodological advantages of sport settings as compared to other trauma research settings (i.e., access to data on premorbid functioning, closely matched control subjects, availability of trauma-related cues, etc.), a sport injury paradigm may be ideal to further research examining psychophysiological response to physical injury and potential implications of physiological reactivity for physical recovery.

The mere presence of posttraumatic distress features, regardless of clinical severity, may have important health implications for athletes. For example, psychological stress-related impairments in immune function may impact athletes' training 
adaptation, risk of and recovery from sport injury; see Clow \& Hucklebridge (2001) for a review. Physiological stress responses have been linked to an impaired ability to fight infection (Cacioppo, 1994; Cacioppo, Malarkey, Kiecolt-Glaser, Uchino, \& et al., 1990; Ironson et al., 1997; Kiecolt-Glaser \& Glaser, 1987; Laudenslager et al., 1998; Petrie, Booth, Pennebaker, Davison, \& Thomas, 1995; Pike et al., 1997), delayed wound healing (Kiecolt-Glaser, Marucha, Malarkey, Mercado, \& Glaser, 1995; Marucha, Kiecolt-Glaser, \& Favagehi, 1998; Whitney \& Heitkemper, 1999), and prolonged recovery from surgery (Jenkins, Langlais, Delis, \& Cohen, 1998; Kiecolt-Glaser, Page, Marucha, MacCallum, \& Glaser, 1998), all of which may impede recovery from sport training and injury. In addition to direct mechanisms, physiological distress may also influence post-injury adaptation and recovery through its indirect influence on health and injury-related behaviors. For example, stress impairs one's ability to self-regulate, which may result in non-adherence to healthy behaviors (e.g., proper nutrition, sufficient sleep, physical activity, abstinence from self-medication) (Heatherton \& Renn, 1995). An inability to selfregulate is also related to treatment non-compliance, where psychological stress and coping behavior has been associated with rehabilitation and recovery outcomes among injured athletes (Brewer et al., 2000; Daly, Brewer, Van Raalte, Petitpas, \& Sklar, 1995; Udry, 1997).

Research regarding posttraumatic stress among athletes with injuries is in its early stages, providing several areas for future research such as those mentioned here. Although no study has explored the efficacy of intervention for athletes' posttraumatic stress response to injury, elements of common treatment modalities for PTSD are very similar to those included in applied sport psychology intervention (i.e., education, exposure, exploration of feelings and beliefs, and coping skills training). Among athlete populations, a handful of studies using psychological interventions (i.e., cognitive-behavioral stress management, stress-inoculation training) are promising in the attempt to reduce distress and limit adverse health outcomes (Johnson, Ekengren, \& Andersen, 2005; Perna et al., 2003; Ross \& Berger, 1996).

\section{Acknowledgments}

This research was supported by a grant awarded to the first author from West Virginia University Academic Affairs and Research.

\section{References}

APA. (2000). Diagnostic and statistical manual of mental disorders, text revision (4th ed.). Washington: American Psychiatric Association.

Andreassi, J.L. (2000). Psychophysiology: Human behavior and physiological response (4th ed.). Mahwah, NJ: Lawrence Erlbaum Associates.

Asendorpf, J.B., \& Scherer, K.R. (1983). The discrepant repressor: Differentiation between low anxiety, high anxiety, and repression of anxiety by autonomic facial verbal patterns of behavior. Journal of Personality \& Social Psychology, 45, 1334-1346.

Baider, L., \& De Nour, A.K. (1997). Psychological distress and intrusive thoughts in cancer patients. Journal of Nervous and Mental Disease, 185, 346-348.

Baum, A. (1990). Stress, intrusive imagery, and chronic distress. Health Psychology, 9, 653-675. 
Baum, A., Cohen, L., \& Hall, M. (1993). Control and intrusive memories as possible determinants of chronic stress. Psychosomatic Medicine, 55, 274-286.

Blanchard, E.B., Hickling, E.J., Barton, K.A., \& Taylor, A.E. (1996). One-year prospective follow-up of motor vehicle accident victims. Behaviour Research and Therapy, 34, 775-786.

Blanchard, E.B., Hickling, E.J., Forneris, C.A., Taylor, A.E., Buckley, T.C., Loos, W.R., et al. (1997). Prediction of remission of acute posttraumatic stress disorder in motor vehicle accident victims. Journal of Traumatic Stress, 10, 215-234.

Brewer, B., Petitpas, A., Van Raalte, J., Sklar, J., \& Ditmar, T. (1995). Prevalence of psychological distress among patients at a physical therapy clinic specializing in sports medicine. Sports Medicine, Training and Rehabilitation, 6, 139-145.

Brewer, B.W., Cornelius, A.E., Van Raalte, J., Petitpas, A., Sklar, J., Pohlman, M. H., et al. (2000). Attributions for recovery and adherence to rehabilitation following anterior cruciate ligament reconstruction: A prospective analysis. Psychology and Health, 15, 283-291.

Brewer, B.W., Linder, D.E., \& Phelps, C.M. (1995). Situational correlates of emotional adjustment to athletic injury. Clinical Journal of Sport Medicine, 5, 241-245.

Brewer, B.W., Van Raalte, J.L., Cornelius, A.E., Petitpas, A.J., Sklar, J.H., Pohlman, M.H., et al. (2000). Psychological factors, rehabilitation adherence, and rehabilitation outcome after anterior cruciate ligament reconstruction. Rehabilitation Psychology, 45, 20-37.

Bryant, R.A., \& Harvey, A.G. (1997). Acute stress disorder: A critical review of diagnostic issues. Clinical Psychology Review, 17, 757-773.

Bryant, R.A., Harvey, A.G., Gordon, E., \& Barry, R.J. (1995). Eye movement and electrodermal responses to threat stimuli in post-traumatic stress disorder. International Journal of Psychophysiology, 20, 209-213.

Cacioppo, J.T. (1994). Social neuroscience: Autonomic, neuroendocrine, and immune responses to stress. Psychophysiology, 31, 113-128.

Cacioppo, J.T., Malarkey, W.B., Kiecolt-Glaser, J.K., Uchino, B.N., \& et al. (1990). Heterogeneity in neuroendocrine and immune responses to brief psychological stressors as a function of autonomic cardiac activation. Psychological Record, 40, 451-457.

Casada, J.H., Amdur, R., Larsen, R., \& Liberzon, I. (1998). Psychophysiologic responsivity in posttraumatic stress disorder: Generalized hyperresponsiveness versus trauma specificity. Biological Psychiatry, 44, 1037-1044.

Clow, A., \& Hucklebridge, F. (2001). The impact of psychological stress on immune function in the athletic population. Exercise Immunology Review, 7, 5-17.

Conn, J.M., Annest, J.L., \& Gilchrist, J. (2003). Sports and recreation related injury episodes in the US population, 1997-99. Injury Prevention, 9, 117-123.

Curry, T.J., \& Strauss, R.H. (1994). A little pain never hurt anybody; a photo-essay on the normalization of sport injuries. Sociology of Sport Journal, 11, 195-208.

Daly, J.M., Brewer, B.W., Van Raalte, J.L., Petitpas, A.J., \& Sklar, J.H. (1995). Cognitive appraisal, emotional adjustment, and adherence to rehabilitation following knee surgery. Journal of Sport Rehabilitation, 4, 23-30.

Davies, M.I., \& Clark, D.M. (1998). Thought suppression produces a rebound effect with analogue post-traumatic intrusions. Behavior Research and Therapy, 36, 571-582.

Davis, P.J. (1990). Repression and the inaccessibility of emotional memories. In J.L. Singer (Ed.), Repression and dissociation: Implications for personality theory, psychopathology, and health (pp. 387-403). Chicago: University of Chicago Press.

Dean, J., Whelan, J., \& Meyers, A.W. (1990, September). An incredibly quick way to assess mood states: The incredibly short POMS. Paper presented at the annual meeting of the Association for the Advancement of Applied Sport Psychology, San Antonio, TX.

Delahanty, D.L., Herberman, H.B., Craig, K.J., Hayward, M.C., et al. (1997). Acute and chronic distress and posttraumatic stress disorder as a function of responsibility for 
serious motor vehicle accidents. Journal of Consulting and Clinical Psychology, 65, 560-567.

Dimsdale, J.E. (2000). Stalked by the past: The influence of ethnicity on health. Psychosomatic Medicine, 62, 161-170.

Fowles, D. (1988). Psychophysiology and psychopathology: A motivational approach. Psychophysiology, 25, 373-391.

Gray, J. (1987). Perspectives on anxiety and impulsivity: A commentary. Journal of Research in Personality, 21, 493-509.

Greenspan, L., McLellan, B.A., \& Greig, H. (1985). Abbreviated Injury Scale and Injury Severity Score: A scoring chart. The Journal of Trauma, 25, 60-64.

Hall, M., Baum, A., Buysse, D.J., Prigerson, H.G., Kupfer, D.J., \& Reynolds, C.F. (1998). Sleep as a mediator of the stress-immune relationship. Psychosomatic Medicine, 60, 48-51.

Harvey, A.G., \& Bryant, R.A. (1998). The effect of attempted thought suppression in acute stress disorder. Behavioral Research \& Therapy, 36, 583-590.

Hayes, S.C., Wilson, K.G., Gifford, E.V., Follette, V.M., \& Strosahl, K. (1996). Experiential avoidance and behavioral disorders: A functional dimensional approach to diagnosis and treatment. Journal of Consulting \& Clinical Psychology, 64, 1152-1168.

Heatherton, T.F., \& Renn, R.J. (1995). Stress and the disinhibition of behavior. Mind-Body Medicine, 1, 72-81.

Heponiemi, T., Keltikangas-Jarvinen, L., Kettunen, J., Puttonen, S., \& Ravaja, N. (2004). BISBAS sensitivity and cardiac autonomic stress profiles. Psychophysiology, 41, 37-45.

Holbrook, T.L., Anderson, J.P., Sieber, W.J., Browner, D., \& Hoyt, D.B. (1998). Outcome after major trauma: Discharge and 6-month follow-up results from the Trauma Recovery Project. The Journal of Trauma, 45, 315-323.

Holmes, D.S. (1990). The evidence for repression: An examination of sixty years of research. In J. L. Singer (Ed.), Repression and dissociation: Implications for personality theory, psychopathology, and health (pp. 85-102). Chicago: University of Chicago Press.

Horowitz, M., Wilner, N., \& Alvarez, W. (1979). Impact of Event Scale: A measure of subjective stress. Psychosomatic Medicine, 41, 209-218.

Ironson, G., Wynings, C., Schneiderman, N., Baum, A., Rodriguez, M., Greenwood, D., et al. (1997). Posttraumatic stress symptoms, intrusive thoughts, loss, and immune function after Hurricane Andrew. Psychosomatic Medicine, 59, 128-141.

Jenkins, M.A., Langlais, P.J., Delis, D., \& Cohen, R. (1998). Learning and memory in rape victims with posttraumatic stress disorder. The American Journal of Psychiatry, 155, 278-279.

Johnson, U., Ekengren, J., \& Andersen, M.B. (2005). Injury prevention in Sweden: Helping soccer players at risk. Journal of Sport and Exercise Psychology, 27, 32-38.

Joseph, R. (1998). Traumatic amnesia, repression, and hippocampus injury due to emotional stress, corticosteroids and enkephalins. Child Psychiatry and Human Development, 29, 169-185.

Kiecolt-Glaser, J.K., \& Glaser, R. (1987). Psychosocial moderators of immune function. Annals of Behavioral Medicine, 9, 16-20.

Kiecolt-Glaser, J.K., \& Glaser, R. (1992). Psychoneuroimmunology: Can psychological interventions modulate immunity? Journal of Consulting and Clinical Psychology, 60, 569-575.

Kiecolt-Glaser, J.K., Marucha, P.T., Malarkey, W.B., Mercado, A.M., \& Glaser, R. (1995). Slowing of wound healing in psychological stress. Lancet, 346, 1194-1196.

Kiecolt-Glaser, J.K., Page, G.G., Marucha, P.T., MacCallum, R.C., \& Glaser, R. (1998). Psychological influences on surgical recovery: Perspectives from psychoneuroimmunology. American Psychologist, 53, 1209-1218.

Kuch, K., Cox, B.J., \& Evans, R.J. (1996). Posttraumatic stress disorder and motor vehicle accidents: A multidisciplinary overview. Canadian Journal of Psychiatry, 41, 429-434. 
Lacey, J.I., Kagan, J., Lacey, B., \& Moss, H.A. (1963). The visceral level: Situational determinants and behavioral correlates of autonomic response patterns. In P.H. Knapp (Ed.), Expression of the Emotions in Man (pp. 122-155). New York: International Universities Press.

Laudenslager, M.L., Aasal, R., Adler, L., Berger, C.L., Montgomery, P.T., Sandberg, E., et al. (1998). Elevated cytotoxicity in combat veterans with long-term post-traumatic stress disorder: Preliminary observations. Brain, Behavior, and Immunity, 12, 74-79.

Lavallee, L., \& Flint, F. (1996). The relationship of stress, competitive anxiety, mood state, and social support to athletic injury. Journal of Athletic Training, 31, 296-299.

Leddy, M.H., Lambert, M.J., \& Ogles, B.M. (1994). Psychological consequences of athletic injury among high-level competitors. Research Quarterly for Exercise and Sport, 65, 347-354.

Llabre, M.M., Spitzer, S.B., Saab, P.G., Ironson, G.H., \& Schneiderman, N. (1991). The reliability and specificity of delta versus residualized change as measures of cardiovascular reactivity to behavioral challenges. Psychophysiology, 28, 701-711.

McNair, D.M., Lorr, M., \& Droppleman, L.F. (1971). Manual for the Profile of Mood States. San Diego: Educational and Industrial Testing Service.

Martin, J.B., \& Pihl, R.O. (1986). Influence of alexithymic characteristics on physiological and subjective stress responses in normal individuals. Psychotherapy \& Psychosomatics, 45, 66-77.

Marucha, P.T., Kiecolt-Glaser, J.K., \& Favagehi, M. (1998). Mucosal wound healing is impaired by examination stress. Psychosomatic Medicine, 60, 362-365.

NCAA. (1996). All sport injury summary. Overland Park, KS.

NCAA. (2005). 1999-00, 2003-04 NCAA student-athlete ethnicity report. Indianapolis, IN.

Newcomer, R., \& Perna, F. (2003). Features of posttraumatic distress among adolescent athletes. Journal of Athletic Training, 38, 163-166.

Newcomer, R., Roh, J., Perna, F., Stilger, V., \& Etzel. (1998). Injury as a traumatic experience: Intrusive thoughts and avoidance behavior associated with injury among college student-athletes. Journal of Applied Sport Psychology, 10, S54.

Nixon, H.L. (1992). A social network analysis of influences on athletes to play with pain and injuries. Journal of Sport and Social Issues, 16, 127-135.

Nixon, H.L. (1993). Accepting the risks of pain and injury in sport: Mediated cultural influences on playing hurt. Sociology of Sport Journal, 10, 183-196.

Nixon, H.L. (1994). Social pressure, social support, and help seeking for pain and injuries in college sports networks. Journal of Sport and Social Issues, 18, 340-355.

Orr, S.P., Lasko, N.B., Metzger, L.J., \& Pitman, R.K. (1997). Physiologic responses to non-startling tones in Vietnam veterans with post-traumatic stress disorder. Psychiatry Research, 73, 103-107.

Peck, D.F., Robertson, A., \& Zeffert, S. (1996). Psychological sequelae of mountain accidents: A preliminary study. Journal of Psychosomatic Research, 41, 55-63.

Perna, F.M., Antoni, M.H., Baum, A., Gordon, P., \& Schneiderman, N. (2003). Cognitive behavioral stress management effects on injury and illness among competitive athletes: A randomized clinical trial. Annals of Behavioral Medicine, 25, 66-73.

Perna, F.M., \& McDowell, S.L. (1995). Role of psychological stress in cortisol recovery from exhaustive exercise among elite athletes. International Journal of Behavioral Medicine, 2, 13-26.

Perna, F., Roh, J., Newcomer, R., Stilger, V., \& Etzel, E. (1998). Clinical depression among injured athletes: An empirical assessment. Journal of Applied Sport Psychology, 10, S54.

Petrie, K.J., Booth, R.J., Pennebaker, J.W., Davison, K.P., \& Thomas, M.G. (1995). Disclosure of trauma and immune response to hepatitis b vaccination program. Journal of Consulting and Clinical Psychology, 63, 787-792. 
Petrie, T.A. (1992). Psychosocial antecedents of athletic injury: The effects of life stress and social support on female collegiate gymnasts. Behavioral Medicine, 18, 127-138.

Petrie, T.A. (1993). Coping skills, competitive trait anxiety, and playing status: Moderating effects on the life stress-injury relationship. Journal of Sport \& Exercise Psychology, 15, 261-274.

Petrie, T.A. (1993). The moderating effects of social support and playing status on the life stress-injury relationship. Journal of Applied Sport Psychology, 5, 1-16.

Pike, J.L., Smith, T.L., Hauger, R.L., Nicassio, P.M., Patterson, T.L., McClintick, J., et al. (1997). Chronic life stress alters sympathetic, neuroendocrine, and immune responsivity to an acute psychological stressor in humans. Psychosomatic Medicine, 59, 447-457.

Prins, A., Kaloupek, D.G., \& Keane, T.M. (1995). Psychophysiological evidence for autonomic arousal and startle in traumatized adult populations. In M.J. Friedman, D.S. Charney \& A.Y. Deutch (Eds.), Neurobiological and Clinical Consequences of Stress: From normal adaptation to post-traumatic stress disorder (pp. 291-314). Philadelphia: Lippincott-Raven.

Purdon, C., \& Clark, D.A. (1994). Obsessive intrusive thoughts in nonclinical subjects. Part ii. Cognitive appraisal, emotional response and thought control strategies. Behaviour Research and Therapy, 32, 403-410.

Roh, J., Newcomer, R., Perna, F., Stilger, V., \& Etzel, E. (1998). Depressive mood states among college athletes: Pre- and post-injury. Journal of Applied Sport Psychology, 10, S54.

Rosenthal, R., \& Rosnow, R. (1988). Contrast Analysis: Focused comparisons in the analysis of variance. New York: Cambridge University Press.

Ross, M.J., \& Berger, R.S. (1996). Effects of stress inoculation training on athletes' postsurgical pain and rehabilitation after orthopedic injury. Journal of Consulting and Clinical Psychology, 64, 406-410.

Schwerdtfeger, A. (2004). Predicting autonomic reactivity to public speaking: Don't get fixed on self-report data! International Journal of Psychophysiology, 52, 217-224.

Shacham, S. (1983). A shortened version of the Profile of Mood States. Journal of Personality Assessment, 47, 305-306.

Shalev, A.Y., Peri, T., Gelpin, E., Orr, S.P., \& Pitman, R.K. (1997). Psychophysiologic assessment of mental imagery of stressful events in Israeli civilian posttraumatic stress disorder patients. Comprehensive Psychiatry, 38, 269-273.

Shiperd, J.C., \& Beck, J.G. (1999). The effects of suppressing trauma-related thoughts on women with rape-related posttraumatic stress disorder. Behavioral Research Therapy, 37, 99-112.

Shuer, M.L., \& Dietrich, M.S. (1997). Psychological effects of chronic injury in elite athletes. Western Journal of Medicine, 166, 104-109.

Stone, A.A. (1997). Measurement of affective response. In S. Cohen, R.C. Kessler \& L.U. Gordon (Eds.), Measuring Stress: A guide for health and social scientists (pp. 148174). New York: Oxford Press.

Trinder, H., \& Salkovskis, P.M. (1994). Personally relevant intrusions outside the laboratory: Long-term suppression increases intrusion. Behavior Research and Therapy, 32, 833-842.

Tunick, R., Etzel, E.F., Leard, J., \& Lerner, B. (1996). Counseling injured and disabled student-athletes: A guide for understanding and intervention. In E.F. Etzel, A.P. Ferrante \& J.W. Pinkney (Eds.), Counseling Student Athletes: Issues and interventions (2nd ed., pp. 157-184). Morgantown, WV: Fitness Information Technology.

Udry, E. (1997). Coping and social support among injured athletes following surgery. Journal of Sport \& Exercise Psychology, 19, 71-90.

Waldstein, S.R., Bachen, E.A., \& Manuck, S.B. (1997). Active coping and cardiovascular reactivity: A multiplicity of influences. Psychosomatic Medicine, 59, 620-625. 
Ward, S.E., Leventhal, H., \& Love, R. (1988). Repression revisited: Tactics used in coping with a severe health threat. Personality \& Social Psychology Bulletin, 14, 735-746.

Warda, G., \& Bryant, R.A. (1998). Thought control strategies in acute stress disorder. Behavior Research and Therapy, 36, 1171-1175.

Wegner, D.A., \& Zanakos, S. (1994). Chronic thought suppression. Journal of Personality, 62, 615-640.

Weinberger, D.A. (1990). The construct validity of the repressive coping style. In J.L. Singer (Ed.), Repression and dissociation: Implications for personality theory, psychopathology, and health (pp. 337-386). Chicago: University of Chicago Press.

Weiss, D.S. (2004). The Impact of Event Scale-Revised. In T.M. Keane \& J.P. Wilson (Eds.), Assessing psychological trauma and PTSD (2nd ed., pp. 168-189). New York: Guilford Press.

Whitney, J.D., \& Heitkemper, M.M. (1999). Modifying perfusion, nutrition, and stress to promote wound healing in patients with acute wounds. Heart Lung, 28, 123-133.

Wickramasekera, I. (1994). Psychophysiological and clinical implications of the coincidence of high hypnotic ability and high neuroticism during threat perception in somatization disorders. The American Journal of Clinical Hypnosis, 37, 22-33.

Yehuda, R., McFarlane, A.C., \& Shalev, A.Y. (1998). Predicting the development of posttraumatic stress disorder from the acute response to a traumatic event. Biological Psychiatry, 44, 1305-1313.

Zilberg, N.J., Weiss, D.S., \& Horowitz, M.J. (1982). Impact of Events Scale: A crossvalidation study and some empirical evidence supporting a conceptual model of stress response syndromes. Journal of Consulting and Clinical Psychology, 50, 407-414. 